\title{
Depressive Symptoms in Danish Elite Athletes Using the Major Depressive Inventory (MDI) and the Center for Epidemiological Studies Depression Scalle (CES-D)
}

Andreas Kuettel*1, Anna Melin ${ }^{2}$, Carsten H. Larsen ${ }^{1}$, \& Mia Beck Lichtenstein ${ }^{1}$

1 Department of Sports Science and Clinical Biomechanics, University of Southern Denmark, Odense, Denmark 2 Department of Sports Sciences, Linnaeus University, Växjö, Sweden

\section{Abstract}

The prevalence of depressive symptoms among athletes is an ongoing debate in the scientific literature. The aim of the current study was to assess the prevalence of depressive symptoms in Danish elite athletes and to evaluate the psychometric properties of the Major Depressive Inventory (MDI) and the Center for Epidemiological Studies Depression Scale (CES-D) in athletes. The total sample comprised 996 athletes from two cross-sectional studies using the MDI ( $n=$ $409)$ and the CES-D ( $n=587)$. Using the original cut-off points, the MDI found $8.6 \%$ and the CES-D found $24.9 \%$ at risk of depression. Using alternative cut-off points, both instruments detected $10-11 \%$ of athletes at risk. No statistically significant differences were found related to age, injury, and type of sport between high risk and low risk groups, whereas female athletes were overrepresented in the high-risk groups. Principal component analyses confirmed a single factor structure in both instruments with sufficient item loadings on the first component and Cronbach $a$ values of .89 and .88. We recommend regular screening of depressive symptoms in elite athletes, with MDI and CES-D as valid instruments for that purpose. The appropriateness of using different cut-off points in athletic samples is discussed.

Key words depression, athletes, sport, assessment, prevalence, cut-off

Recent research has indicated that elite athletes are at risk of mental health problems in comparable ways as the general population (Fernandes et al., 2019; Gorczynski, Coyle, \& Gibson, 2017). However, elite athletes are exposed to unique challenges and stressors including demands of competition and training, dealing with injury and overtraining, and expectations from public and sponsors that may influence their mental health state (Arnold \& Fletcher, 2012; Kuettel \& Larsen, 2019). A greater risk of mental disorders may be experienced by elite athletes who are injured, approaching/in retirement, or experiencing performance difficulty (Rice et al., 2016). Most of the studies investigating mental health disorders in athletes have focused on mood disorders such as depression and anxiety (Golding et al., 2020; Rice et al., 2019), while other studies have investigated eating disorders (Sundgot-Borgen \& Torstveit, 2004), problems related to sleep, substance abuse, or addiction disorders (Gouttebarge et al., 2015; Reardon et al., 2019; Szabo et al., 2013).

\section{Depression in Elite Sport}

Depression is a widely used term that describe a person's mood as being reduced from what is perceived as being normal (American Psychiatric Association, 2013; Baron et al., 2013). Depressive symptoms such as reduced self-confidence and hopelessness are among the most identified mental health issues among athletes (Reardon et al., 2019). However, the current evidence varies widely in the reported prevalence rates, with proportions of moderate or severe symptoms ranging from $3.7 \%$ to $36.5 \%$ in high-performance athletes (Gorczynski et al., 2017). This divergence can mostly be explained by the different self-reporting tools being used in these studies (for an overview, see Tahtinen, Shelley, \& Morris, 2021). In one of the few studies using clinical interviews based on the Diagnostic and Statistical Manual of Mental Disorders (DSM-5), Hammond et al. (2013) found that 34\% of Canadian elite swimmers reported symptoms consistent with a depressive disorder. In a Danish context, Jensen et al. (2018) found a prevalence of $16.7 \%$ among football players, while Kuettel, Pedersen, and Larsen (2020) found a prevalence of $21.1 \%$ in athletes from 18 different sports (both team and individual) reporting moderate or severe symptoms. Both studies used the Epidemiologic Studies Depression Scale (CES-D; Radloff, 1977). In general, and similar to research of normative gender differences (Breslau et al., 2017; Salk, Hyde, \& Abramson, 2017), female athletes have been shown to report higher levels of depressive symptoms than male athletes (Gorczynski et al., 2017, Schaal et al., 2011).

How to Measure Depressive Symptoms in Athletes? Researchers in different countries (most studies were conducted in Northern America and in Europe) investigating depression in athletes have mainly used the CES-D (Radloff, 1977). The Patient Health Questionnaire (PHQ-9; Spitzer, Kroenke, \& Williams, 1999), Beck Depression Inventory (BDI; Beck et al., 1961) and the Major Depression Inventory (MDI; Bech et al., 2001) have also been used although all these scales were developed for 
the general population. The MDI has been used assessing depressive symptoms in regular exercisers (Lichtenstein et al., 2017) and showed good psychometric propertied compatible with the DSM-5 criteria for depression. Notably, even when using a particular tool for screening for depression (such as the CES-D), scholars have used different cut-off points to determine depressive symptoms in athletic samples (Golding et al., 2020). To illustrate, for the CES-D, most studies with athletes have used the conventional cut-off point $>16$ to classify athletes according to their symptomatology (e.g., Beable et al., 2017, Gulliver et al., 2015, Kuettel et al., 2020), whereas other studies have used a cut-off point >22 (e.g., Nixdorf et al., 2013). Cuijpers, Bolujit, and van Straten (2008) attempted to validate both the CES-D and the MDI in an online survey with a sample consisting of 1932 Dutch non-athletic adolescents, followed by an International Neuropsychiatric Interview of 242 participants to determine the presence or absence of a depressive disorder. They found the best cutoff values (i.e., highest sensitivity and specificity measures) to be 22 for the CES-D (considerably higher than the cutoff 16 suggested by Radloff, 1977) and 19 for the MDI (slightly lower than the cut-off 20 proposed by Bech et al., 2011).

Overall, sport-specific assessment tools detecting mental disorders in elite athletes remain scarce (Henriksen et al., 2019). As noted by Larsen et al. (2021), most tools have not been widely used and tested in the sport context yet and the results from the use of these measures should be interpreted with caution. Traditional screening tools that are extensively used and validated with the general population to detect sub-clinical and clinical symptoms of anxiety and depression bear limitations when used in elite sport contexts (Henriksen et al., 2019). For example, precompetition anxiety or the post-Olympic void are common among many high-performance athletes (Reardon et al., 2019). As such, athletes may obtain 'false positive' results when using a general anxiety or depression screening tool applying traditional cut-off points.

\section{Depressive Symptoms Related to Type of Sports}

Identifying the context and reporting findings of depression and anxiety in specific sports is important as this can further improve our understanding of the potential sport-specific variables that need to be considered in future prevention efforts (Tahtinen et al., 2021). Coaches and sport organizations are often curious whether "their" athletes thrive better or worse than athletes from other sports (Bisset, Kroshus, \& Hebard, 2020). Recent evidence has shown that vulnerability to depressive symptoms may vary across different types of sports (Wolanin et al., 2016). However, as discussed by several authors (e.g., Tahtinen et al., 2020; Wolanin et al., 2016), samples have often consisted of a relatively small number of athletes across different sports, and therefore, comparisons have tended to focus on differences between broader sport categories, such as team and individual sports (Beable et al., 2017; Nixdorf et al., 2013). Researchers have underlined that future studies should include larger samples across specific sports to better understand potential sport-specific differences related to athletes' mental health (Kuettel \& Larsen, 2019). To overcome the small-samples-size dilemma in elite sport, Schaal et al. (2011) categorized French elite athletes into seven broader categories (i.e., aesthetic-, fine-motor skills-, racing-, racket-, contact-, team ball-, and high-risk sports) and found that especially aesthetic- and contact sports had a higher prevalence of depression compared with the other sport categories. Other researchers (e.g., Niebauer et al., 2018) have categorized sports in more simple categories (e.g., skill, power, endurance, and mixed sports) related to the cardiac intensity.

Given the variation of depressive symptoms in athletes reported in previous studies (Gorczynski et al., 2017) and the potential influence of the type of sport for athletes' depression discussed in the literature (Wolanin et al., 2016) as well as ongoing public debate in the national Danish media (e.g., Weekendavisen, 2020), the current study aimed to provide contextualized evidence concerning Danish athletes' depressive symptoms and to discuss the appropriateness of using common cut-off points in athletic samples.

\section{Aims}

The main aim of the study was to assess the prevalence of depressive symptoms in Danish elite athletes with the MDI and the CES-D using different cut-off points. A secondary aim was to conduct a brief psychometric evaluation of both scales in these athletic samples. The third aim was to characterize athletes with higher risk of depression according to age, gender, exercise volume, injury profile, and type of sports.

\section{Materials and Methods}

\section{Research Design}

This study was part of two larger cross-sectional research studies conducted with Danish elite athletes. Kuettel et al.'s (2020) study assessed athletes' mental health profiles and factors (e.g., social support, sport environment, stressors) affecting mental health states, whereas Lichtenstein et al.'s (2021) study was mainly focusing on eating disorder symptoms and exercise addiction. The two studies used two different screening scales of depressive symptoms, namely the CES-D and the MDI. In both studies, data were collected in collaboration with the National Olympic Committee and Sports Confederation of Denmark (DIF) and Team Denmark that provided access to elite athletes at the highest national level. To be eligible for the study, athletes had to (a) be part of a national youth or elite sports program, and (b) participate in international competitions or the highest national level. Included athletes were contacted via their sport federation and subsequently received a link to the online survey. For further analyzes and comparison, we categorized all participants into five broader sports categories based on the typology of Niebauer et al. (2018) since there were no athletes from racing or high-risk sports in our samples (as in the study of Schaal et al., 2011). This categorization was also meaningful in terms of achieving group sizes with minimum 20 athletes per type of sport in both samples (see Table 1 ).

\section{Ethics and GDPR}

The recruitment procedure in both studies met the standards of the European general data protection regulations (GDPR) and safeguarding privacy rights of sensitive personal data. Conversely, this procedure did not allow calculation of the probability and response rates, being a not-list-based survey (Bryman, 2012). The Regional Committee on Health Research Ethics for Southern Denmark (Ref. nr. 20182000-107; 20192000145 , respectively) confirmed that anonymous questionnaire studies did not need ethical approval. All participants (or parents for participants aged 15-17 years) gave written consent to participate in the study. Participants were informed that participation was 
voluntary, that no individual could be identified, and that their information will only be used for research purposes.

\section{Data Collection MDI}

\section{Participants}

The study sample consisted of 409 Danish elite athletes from 15 different sports (209 female athletes, 20.4 \pm 5.0 years and 200 male athletes, $19.6 \pm 4.4$ years). The data collection ran from December 2019 to March 2020. The majority of the sample $(45.0 \%)$ was involved in endurance sport (e.g., running, cycling, orienteering, rowing, triathlon, swimming); $21.6 \%$ in team ball sports such as handball and football; $21.4 \%$ in power sports (e.g., karate, wrestling, boxing); $7.0 \%$ in skills sport (e.g., gymnastics, sports dance); and further $5.0 \%$ were racket sports athletes (e.g., badminton). Eleven athletes did not specify their disciplines.

\section{Measures}

Participants completed the MDI (Bech et al., 2001) that has 10 items rated on a 6-point Likert scale from 0 (no time) to 5 (all the time) within the previous two weeks. The total score ranges from 0 to 50, where a higher score reflects more symptoms of depression. Recommended cutoff points (Bech et al., 2015) are 21 for mild depression, 26 for moderate depression, and 31 for severe depression. We report both prevalence of original cut-off for MDI (score of $\geq 21$ to indicate 'symptoms of depression', comprising mild, moderate, and severe depression) and prevalence related to the cut-off points score of $\geq 20$ recommended by Cuijpers et al. (2008) with a sensitivity of $90.5 \%$ and a specificity of $71.5 \%$. Participants also provided demographics (age, gender), information about their trainings load per week, as well as amounts of serious injuries in the past year (defined as the number of time loss from training and competition > 28 days; Bahr et al., 2020).

\section{Data Collection CES-D}

\section{Participants}

The study sample consisted of 587 Danish elite athletes from 18 different sports ( 247 female athletes, 20.0 \pm 4.7 years and 340 male athletes, $18.3 \pm 3.8$ years), distributed between endurance sports (14.5\%), ball sports (e.g., basketball, handball, football, volleyball: 62.6\%), endurance sports (e.g., cycling, rowing, triathlon: 14.5\%), racket sports (e.g., badminton, tennis: 9.0\%), skills sport (e.g., golf, sailing, sports dance: $7.5 \%$ ), power sports (e.g., karate, wrestling: $6.8 \%)$, and racket sports $(9.0 \%)$. The data collection ran from October 2018 to February.

\section{Measures}

Participants completed the Center for Epidemiological Studies Depression scale (CES-D; Radloff, 1977 ) which is a commonly used reliable and valid 20 -items tool to measure depressive symptoms. Statements such as "I felt awful", "People were unfriendly", "I talked less than usual" were answered on a scale from 0 (rarely or none of the time) to 3 (most or all of the time) for how often athletes felt this way during the last week. Four items are stated positive (e.g., "I was happy", "I enjoyed life") and were reverse coded for the analyses, so that higher values generally indicate higher symptomatology. The original cutoff points (Radloff, 1977) related to symptomatology are 0 15 (no to mild), 16-23 (moderate), and 24-60 (severe). Usually, a CES-D score of $\geq 16$ is used to indicate 'symptoms of depression' (thus comprising moderate and severe depression). At this threshold, the CES-D was found to have a sensitivity of $86 \%$ and a specificity of $90 \%$ (Radloff, 1977). The study by Cuijpers et al. (2008) recommended using a cut-off point at score of $\geq 23$ to achieve higher psychometric qualities with a sensitivity of $90.5 \%$ and a specificity of $74.3 \%$. Participants also provided demographics and information about their trainings load and past injuries.

As mentioned earlier, data collection was anonymous in both. When looking at the participants divided by sports discipline, we assume that there is a potential overlap of athletes in 10 disciplines, most of them classified as either endurance or ball sports (athletics [running], badminton, bicycle, football, handball, rowing, orienteering, sports dance, swimming, and triathlon; $76,8 \%$ of the total sample). Within these disciplines, $65 \%$ of the athletes may have participated in both studies (e.g., there were 40 athletes from athletics in the CES-D and 53 in the MDI study). Hence, there was a $50 \%(n=498)$ potential overlap of athletes participating in both studies. Athletes from golf, basketball, ice hockey and volleyball were only represented in the sample using the CES-D, and athletes from boxing, gymnastics, and karate were only part of the MDI sample. 
Table 1: Characteristics and Depressive Symptoms of the Participants in the MDI and CES-D study

\begin{tabular}{llll}
\hline & MDI-study $(n=409)$ & CES-D-study $(n=587)$ & $p$-value \\
\hline Gender (females / males) & $51.1 \% / 48.9 \%$ & $42.2 \% / 57.8 \%$ & .005 \\
Age, years & $20.0 \pm 4.7$ & $19.0 \pm 4.3$ & .001 \\
Training hours/week & $15.8 \pm 6.1$ & $14.8 \pm 6.9$ & .043 \\
Number of injuries in the last year & $1.6 \pm 1.0$ & $1.3 \pm 1.1$ & .001 \\
Type of sport & & & .001 \\
$\quad$ Skills sports & $n=28(7.0 \%)$ & $n=44(7.5 \%)$ & $n=40(6.8 \%)$ \\
$\quad n=85(21.4 \%)$ & $n=365(62.2 \%)$ & $n=85(14.5 \%)$ \\
$\quad$ Power sports & $n=86(21.6 \%)$ & $n=53(9.0 \%)$ & NA \\
$\quad$ Endurance sports & $n=179(45.0 \%)$ & & .001 \\
$\quad$ Raquet sports & $n=20(5.0 \%)$ & $11.3 \pm 8.4(0-60)$ & \\
Depressive symptoms & & $75.1 \%(0-15)$ & \\
$\quad$ Total score (range) & $9.0 \pm 7.3(0-50)$ & - & \\
$\quad$ No depression (range) & $91.4 \%(0-20)$ & $8.5 \%(24-60)$ & \\
$\quad$ Mild depression (range) & $3.9 \%(21-25)$ & $10.7 \%(23-60)$ & .657 \\
$\quad$ Moderate depression (range) & $2.0 \%(26-30)$ & $2.7 \%(31-50)$ & \\
$\quad$ Severe depression (range) & $10.0 \%(20-50)$ & & \\
$\quad$ Cut-off 19 (MDI) and 22 (CES-D) & . & & \\
\hline
\end{tabular}

Note. Numerical data are presented as Mean \pm SD. Categorization of symptomatology refer to Bech et al. (2001) for the MDI and Radloff (1977) for the CES-D. Cut-off 19 (MDI) and 22 (CES-D) according to the study of Cuijpers et al. (2008). $p$-values refer to $t$-tests for continuous variables (age, training hours and injuries) and Chi-square tests for categorical variables (gender, type of sport, symptomatology). NA = not available because of different instrument.

\section{Statistical Analyses}

The following analyses were conducted in IBM SPSS version 26

1. Descriptive analyses to establish the different cut-off points for the MDI and CES-D.

2. Principal component analyses (PCA) to test the factor structure and item loadings of the two scales.

3. Cronbach a to assess the internal reliability of the scales.

4. Chi-square and t-test were used to compare the MDI and CES-D groups and/or sport category groups.

\section{Results}

Despite the potential overlap of participants in both samples, the results showed that there were relatively more female athletes in the MDI study compared with the CES-D study $(51.1 \%$ vs. $42.2 \%, p=.005)$. Athletes participating in the MDI study were on average one year older $(\mathrm{p}=.001)$, trained slightly more per week $p=.043)$, and were significantly more often injured $(p=.001)$ in the past year than the athletes in the CES-D study (see Table 1 ). There was also a significant difference in the distribution in terms of type of sport, with more athletes in the MDI study doing power or endurance sports, whereas there was a larger proportion of ball sports athletes in the CES-D study.
Regarding depressive symptoms, $8.6 \%$ in the MDI sample expressed mild, moderate, or severe symptoms of depression ( $12.4 \%$ female and $4.5 \%$ male) when using the traditional cut-off $\geq 21$, while the CES-D categorized $24.9 \%$ ( $30.7 \%$ female and $20.6 \%$ male) of the sample with either moderate or severe depressive symptoms when using the conventional cut-off $\geq 16$. Categorizing depressive symptomatology in three categories in both scales (i.e., nomild; moderate; severe) using the conventional cut-off points, a statistically significant difference was found between the two samples concerning symptomatology $\left(X^{2}\right.$ $=73.05, d f=2, p<.001$, Cramer's $\mathrm{V}=0.27)$. When using the cut-off points (i.e., $\geq 20$ for MDI and $\geq 23$ for the CESD) proposed by Cuijpers et al. (2008), the prevalence of both scales was $10-11 \%$ to detect athletes with depressive symptoms $(14.8 \%$ females and $5 \%$ males for the MDI, $14.6 \%$ females and $7.9 \%$ males for the CES-D, respectively), yielding no statistical difference regarding symptomatology between the two samples of the MDI and the CES-D study $\left(X^{2}=.19, d f=1, p<.66\right.$, Cramer's $\mathrm{V}=$ $.01)$. The distributions of scores for the MDI (M = 9.0, SD $=7.3)$ and the CES-D $(M=11.3, S D=8.4)$ are depicted in Figure 1 and 2, showing left-skewed curves for both scales. Both original cut-offs (solid line) and the cut-offs proposed by Cuijpers et al. (2008) (dashed line) are delineated. 
Figure 1: Distribution of Scores Using the Major Depression Inventory $(n=409)$

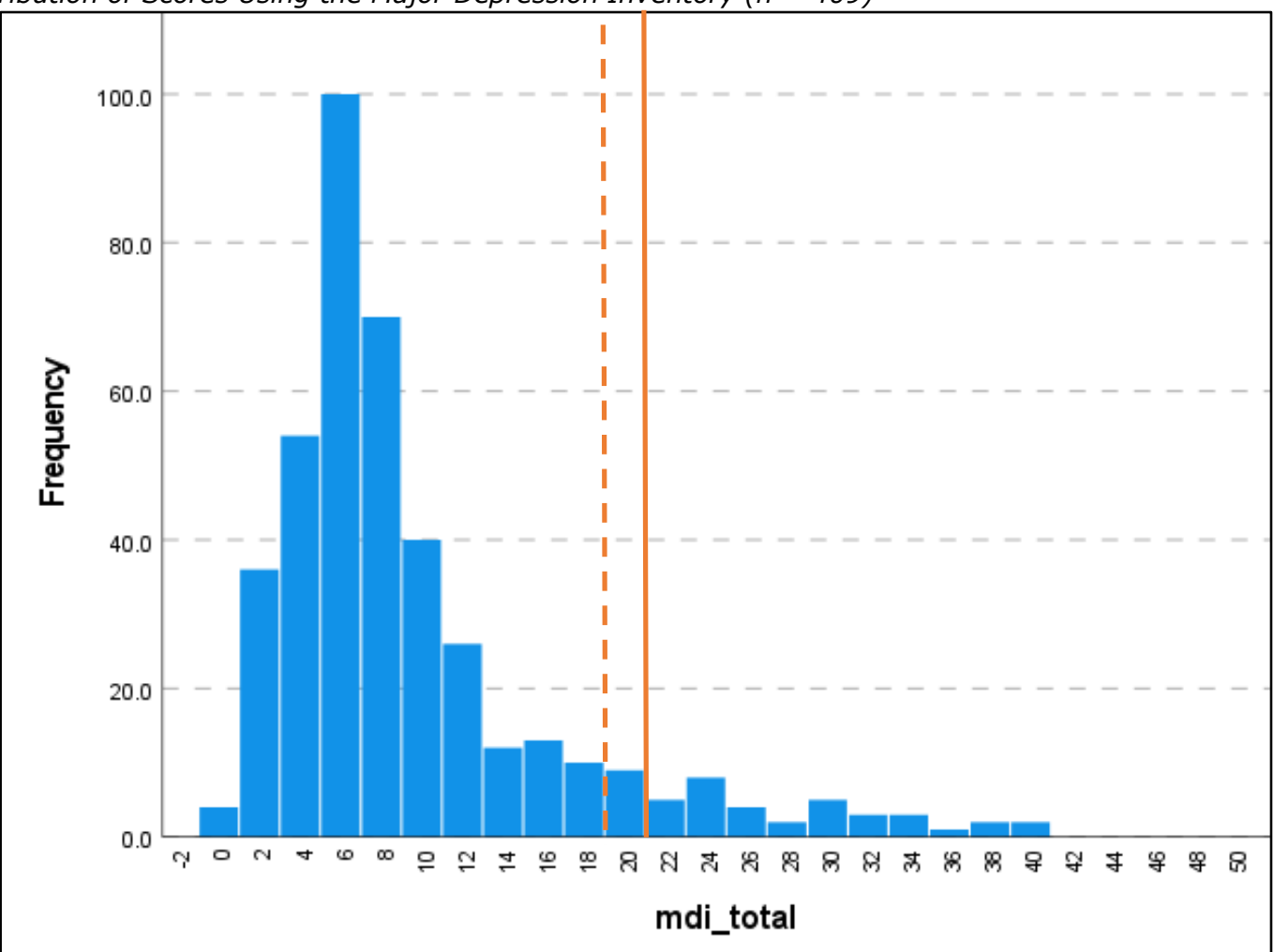

Note. The solid line represents the original cut-off at 21 (Bech et al., 2001) and the dashed line is the cut-off at score 19 proposed by Cuijpers et al. (2008)

Figure 2: Distribution of Scores Using the Center for Epidemiological Studies Depression Scale $(n=587)$

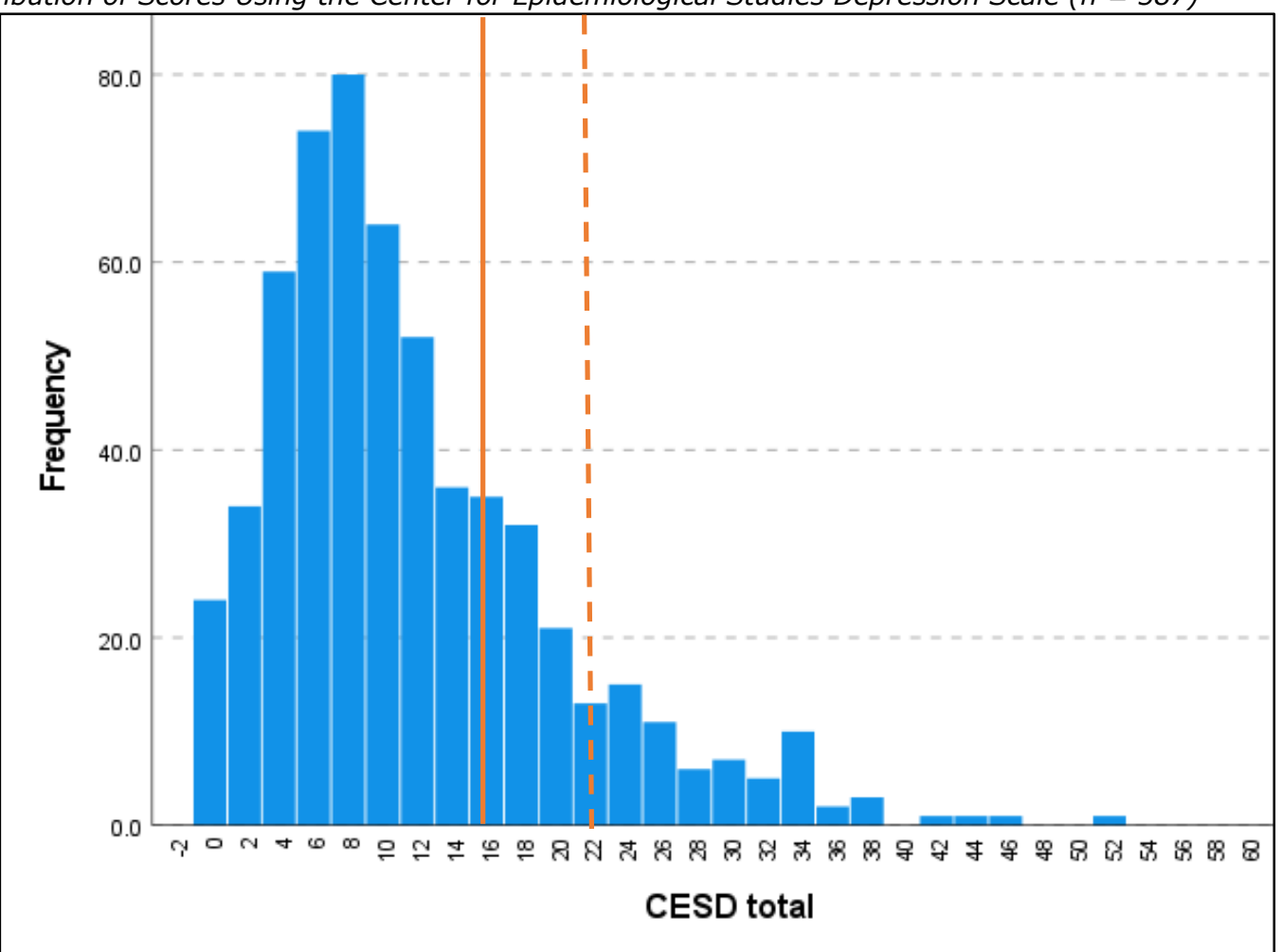

Note. The solid line represents the original cut-off at 16 (Radloff, 1977) and the dashed line is the cut-off at score 22 proposed by Cuijpers et al. (2008). 
Psychometric Evaluation of the MDI and the CES-D

We conducted a PCA for both scales and found evidence for a single factor structure for MDI and CES-D. For the MDI, $54.5 \%$ of the variance was explained by the first factor with an eigenvalue of 5.5. Factor loadings were between 0.85 and 0.51 . Adding a second factor only contributed with further $10 \%$ of the variance. For the CES$D, 32.8 \%$ of the variance was explained with the first factor with an eigenvalue of 6.6. Factor loadings were between 0.75 and 0.36 , with two items loading slightly lower than the recommended 0.40 by DeVellis (2017). Adding a second factor only contributed with further $6.8 \%$ of the variance. See Appendix for more detailed information about the PCA. The Cronbach $a$ coefficients in the two studies were excellent with 0.89 for the MDI and 0.88 for the CESD.

Description of Athletes with High Versus Low Risk of Depressive Symptoms using the MDI and the CES-D Using the cut-off $\geq 20$ for MDI, a significant difference in the gender distribution was detected by chisquare tests indicating that female athletes were overrepresented in the high-risk depression group $\left(x^{2}=\right.$
10.96, $d f=1, p<.001$, Cramer's V = .16). No statistically significant differences in age $(p=.27)$, exercise volume ( $\mathrm{p}$ $=.68)$, number of injuries $(p=.19)$, or type of sport $(p=$ .23 ) between athletes above or below the cut-off point for depressive symptoms were found, even though athletes in the high-risk group were slightly older, had more training hours per week, and reported more injuries in the past (see Table 2).

Using the cut-off point $\geq 23$ for the CES-D, more female than male athletes were categorized being in the high-risk depression group $\left(x^{2}=6.57, d f=1, p<.01\right.$, Cramer's $\mathrm{V}=.11)$. As with the MDI, there were no differences in terms of age $(p=.79)$, exercise volume ( $p=$ $.44)$, injury history $(p=.35)$, or the type of sport category $(p=.11$ ) between the high-risk versus low-risk depression group (see Table 3 ). When conducting the same analyses for the MDI and the CES-D with the traditional cut-offs, results with similar $p$-values (not reported here for reasons of redundance) were obtained within both samples

Table 2: Characteristics of Athletes with High Risk Versus Low Risk of Depression According to the MDI Cut-Off Point 19.

\begin{tabular}{|c|c|c|c|}
\hline & $\begin{array}{l}\text { High risk of depression }(20-50) \\
(n=41)\end{array}$ & $\begin{array}{l}\text { Low risk of depression }(0-19) \\
(n=368)\end{array}$ & $p$ \\
\hline Females / males & $14.8 \%(n=31) / 5.0 \%(n=10)$ & $85.2 \%(n=178) / 95 \%(n=190)$ & .001 \\
\hline Age, years & $20.8 \pm 4.7$ & $19.9 \pm 4.8$ & .271 \\
\hline Training h/week & $16.2 \pm 5.9$ & $15.7 \pm 6.2$ & .678 \\
\hline Injuries & $1.8 \pm 0.9$ & $1.6 \pm 1.0$ & .195 \\
\hline Type of sport & & & .233 \\
\hline Skill & $0.0 \%(n=0 / 28)$ & $100 \%(n=28 / 28)$ & \\
\hline Power & $14.1 \%(n=12 / 85)$ & $85.9 \%(n=73 / 85)$ & \\
\hline Team/Ball & $8.1 \%(n=7 / 86)$ & $91.9 \%(n=79 / 86)$ & \\
\hline Endurance & $10.1 \%(n=18 / 179)$ & $89.9 \%(n=161 / 179)$ & \\
\hline Raquet & $15.0 \%(n=3 / 20)$ & $85.0 \%(n=17 / 20)$ & \\
\hline
\end{tabular}

Note: Numerical data are presented as Mean $\pm S D$. $p$-values refer to $t$-test for continuous variables (age, training hours, injuries) and Chi-square test for categorical variables (gender, type of sport).

Table 3: Characteristics of Athletes with High Risk Versus Low Risk of Depression According to the CES-D Cut-Off Point 22.

\begin{tabular}{|c|c|c|c|}
\hline & $\begin{array}{l}\text { High risk of depression }(23-60) \\
(n=63)\end{array}$ & $\begin{array}{l}\text { Low risk of depression }(0-22) \\
(n=524)\end{array}$ & $p$ \\
\hline Females / males & $14.6 \%(n=36) / 7.9 \%(n=27)$ & $85.4 \%(n=211) / 92.1 \%(n=313)$ & .010 \\
\hline Age, years & $19.1 \pm 3.3$ & $18.9 \pm 4.4$ & .789 \\
\hline Training h/week & $15.5 \pm 8.1$ & $14.8 \pm 6.7$ & .439 \\
\hline Injuries & $1.4 \pm 1.0$ & $1.3 \pm 1.2$ & .346 \\
\hline Type of sport & & & .106 \\
\hline Skill & $11.4 \%(n=5 / 44)$ & $88.6 \%(n=39 / 44)$ & \\
\hline Power & $12.5 \%(n=5 / 40)$ & $87.5 \%(n=35 / 40)$ & \\
\hline Team/Ball & $11.2 \%(n=41 / 365)$ & $88.8 \%(n=324 / 365)$ & \\
\hline Endurance & $14.1 \%(n=12 / 85)$ & $85.9 \%(n=73 / 85)$ & \\
\hline Raquet & $0.0 \%(n=0 / 53)$ & $100 \%(n=53 / 53)$ & \\
\hline
\end{tabular}

Note: Numerical data are presented as Mean $\pm S D$. $p$-values refer to $t$-test for continuous variables (age, training hours, injuries) and Chi-square test for categorical variables (gender, type of sport). 


\section{Discussion}

\section{Prevalence of Depressive Symptoms in Danish Elite} Athletes

Using the traditional cut-off points, the prevalence of moderate to severe depressive symptoms in Danish elite athletes from a variety of different sports was $8.6 \%$ using the MDI, and $24.9 \%$ using the CES-D, respectively. The difference in the prevalence proportions between the two samples comprising of Danish elite athletes with similar characteristics related to gender, age, and exercise volume was unexpectedly high. However, when using the recommended cut-offs from Cuijpers et al. (2008) (i.e., $>19$ for MDI and >22 for CES-D), we found similar prevalence rates for both screening tools with $10 \%$ (MDI) and $10.7 \%$ (CES-D) being at risk of depression. Based on these findings and the comparison of both scales, we propose to adjust the conventional cut-off for the CES-D to $>22$ in athletic samples (which is close to the original cutoff $>23$ related severe symptoms) to avoid over-classifying athletes experiencing depressive symptoms. Reporting that every tenth instead of every forth athletes experience depressive symptoms depicts a quite different picture, and this might impact how mental health of elite athletes is perceived and debated in public, and how sport federations rethink and adjust their initiatives to work with issues around athletes' mental health (Henriksen et al., 2019). It has been underlined that the optimal cut-off score depend on the setting in which assessment is conducted, meaning that the same cut-off point can result in many false positives in one setting while leading to more false negatives in another (Manea, Gilbody, \& McMillan, 2012). We must acknowledge that we cannot give a final answer to which, when, and why the cut-off points for the two scales are adequate in athletic samples. For that, a diagnostic follow-up interview with athletes that score around the cut-off points should be conducted. We suggest that future studies further validate the cut-off points of both scales in athletic samples, also from a gender perspective. Current review studies (Golding et al., 2020; Tahtinen et al., 2021) concluded that the prevalence of depressive symptoms in high-performance athletes varied from $6.7 \%$ to $34.0 \%$, mainly based on CES-D studies, and our findings align well with these numbers. As suggested by Tahtinen et al. (2021), we reported not only single cutoffs for the scales but also prevalence rates across different levels of severity along with histograms to provide a more transparent perspective of the symptom prevalence in athletes. Around one in ten Danish elite athletes in our study reported depressive symptoms, but these symptoms were not related to age, injury status, or type of sport in our samples. A Danish study (Lichtenstein et al., 2017) measured depressive symptoms with the MDI in recreational exercisers from ball sport (e.g., football, handball), endurance sports (e.g., running, cycling, swimming,), strength sport (e.g., fitness, weightlifting), aesthetic sports (dancing and gymnastics), and racket sport. Using the MDI cut-off point $\geq 26$, the study found that $5 \%$ of the recreational exercisers reported symptoms of depression while it was the case for $12 \%$ of the participants with orthopedic injuries. No association between depressive symptoms and injury duration or number of previous injuries was found. A similar study (Lichtenstein et al., 2018) reported prevalence proportions using MDI in regular exercisers with high risk of exercise addiction and present injury (26\%) and without present injury (13\%) using the cut-off $\geq 26$. However, both studies would have found higher prevalence when using cut-off point $\geq 21$ for mild depression, indicating that elite athletes do not report higher depression scores than recreational exercisers. The current study identified $4.7 \%$ of the elite athletes as having moderate to severe depressive symptoms. Based on these comparisons, elite athletes do not seem to have a higher risk of depressive symptoms than recreational exercisers.

Psychometric Properties of the MDI and CES-D in Athletic Samples

The PCA confirmed that all items had acceptable loadings on the first factor in both scales, and the first factor contributed significantly to the explained variance. Our brief reliability checks of the scales indicated high internal stability with Cronbach $a$ values of 0.89 and 0.88 , respectively, are in line with Cuijpers et al. (2008) who found Cronbach $a$ values at 0.88 and 0.93 for the scales, respectively. Due to the design of the current study, we were not able to assess the construct validity of the scales, but Cuijpers found that MDI and CES-D correlated highly with each other (Pearson's $r=.88 ; p<.001$ ) indicating that both scales identify the same underlying construct, namely depressive symptoms. Thus, it could be argued that both tools are suitable when screening for depressive symptoms in elite athlete populations. The MDI and CES-D may be useful scales, but MDI might be easier to administer since it only has 10 items compared to CES-D that has 20 items.

\section{Athletes' Risk Profiles for Depression}

Our findings confirm previous research (Gorzyinski et al., 2017; Golding et al., 2020) that female athletes are more prone to express moderate or severe depressive symptoms compared to male athletes, independent of the scale or the cut-off points used. This is also the case in the general population, where multiple factors (e.g., hormonal and neuro-developmental changes during puberty, income inequality, discrimination, cultural influences) were identified as pathways to the gender difference in depression (Salk et al., 2017). Athletes in the high- and low-risk groups did not differ in terms of age and amount of training hours or injuries, highlighting that depressive symptoms appear across age and athletic level, and team/individual sports. When examining Danish athletes with different mental health profiles, Kuettel et al. (2020) found that flourishing athletes experienced better social support and expressed fewer stressors than athletes with higher symptomatology of depression and anxiety. However, it is generally acknowledged that there are many factors (e.g., within-individual differences, social-, interpersonal, and environmental factors; Kuettel \& Larsen, 2019; Tahtinen et al., 2021) that influence depressive symptoms. Therefore, it is unlikely that a single causal pathway or mechanism can ever fully explain the development, maintenance, and/or recurrence of depression (Hankin, 2012).

This study divided athletes from a variety of sport disciplines into five broader types of sports according to the typology of Niebauer et al. (2018). Compared to Schaal et al. (2011) who found differences between aesthetic and other sports, our study did not detect significant differences when comparing type of sports in relation to high and low risk of depression using the cut-off point of Cuijpers et al. (2008). Applying a multifactorial perspective (Hankin, 2012) described above, being engaged in a certain sport discipline (environmental factor) cannot per se explain why some athletes experience depressive symptoms and some not. However, some interesting findings were detected in the two samples. In the MDI group, high risk of depression varied from $0 \%$ (skill sports) to $15 \%$ (racket sports), whereas in the CES-D group, high risk of depression ranged from $0 \%$ (racket sports) to $14.1 \%$ (endurance sport). 
These findings highlight that more research with larger samples within the sub-groups is needed to make more precise statements about the potential influence of type of sport on depressive symptoms. When analyzing the pooled sample from the two studies measured with the MDI and the CES-D, we can conclude that there are athletes in all sport types that express symptoms that refer to a moderate depression. Hence, periodical regular screening (both in and off-season) of depressive symptoms in elite athletes in all sports is recommended, since early detection may prevent severe courses and contributes to reduce stigmatization of mental health issues in athletes (Purcell et al., 2019).

\section{Limitations}

It is a major limitation of the study design that the two screening tools for depression were not assessed in the same sample. We recommend future studies to use the MDI and the CES-D in the same sample to conduct a more valid and robust psychometric evaluation. The research design made it not possible to conclude how many athletes were screened with both tools, but a certain overlap (maximum $50 \%$ ) can be expected due to the similar number of athletes representing a sports discipline. The two different tools found almost the same prevalence rates and factor structure despite different samples. Furthermore, a diagnostic interview should be added to address sensitivity and specificity of the scales in elite athletes. Values are based on athletes' self-reporting without information about the response rate, and therefore, a potential (non)respondent bias cannot be excluded, given the sensitivity of the topic and the general stigma about mental health in elite sport (Castaldelli-Maia et al., 2019). Our study included only gender, age, injury, training load, and sports type as variables that potentially influence depression in athletes and neglected to look at many of the other risk and protective factors for athletes' mental health (Kuettel \& Larsen, 2019). Furthermore, we did not collect data in a non-athletic control group to compare prevalence proportions. To date, this is the largest Danish study exploring depressive symptoms in nearly 1000 elite athletes, making it possible to categorize athletes into different type of sports and compare them according to depressive symptoms.

\section{Conclusions}

This study found that using the original cut-off points for depression, the MDI categorized $8.6 \%$ and the CES-D $24.9 \%$ of the Danish athletes at risk of depression. However, when using alternative recommended cut-off points, we found that $10-11 \%$ of athletes in both samples expressed symptoms of moderate to severe depression. The internal reliability of the scales was high in both samples and the principal component analyses confirmed a single factor structure in both scales with sufficient item loadings at the first factor. We found no significant difference in age, injury history, and type of sport, while female gender seemed to be a risk factor for depressive symptoms. We recommend regular screening of depressive symptoms in elite athletes with either the MDI (using cutoff 19) or the CES-D (using cut-off 22). The MDI may be easier to administer because of its briefness. Future studies should compare the two tools within the same sample and further assess the validity of the cut-off points in athletic samples with clinical follow-up interviews.

\section{Acknowledgments}

We would like to express our gratitude to Team Denmark and the National Olympic Committee and Sports Confederation of Denmark (DIF) for supporting both projects, and to Lars Holm for providing feedback on earlier drafts of the manuscript.

\section{References}

American Psychiatric Association (2013). Diagnostic and statistical manual of mental disorders ( $5^{\text {th }}$ ed). Washington, DC. https://doi.org/10.1176/appi.books.9780890425596

Bahr, R., Clarsen, B., Derman, W., Dvorak, J., Emery, C. A., Finch, C. F., ...Chamari, K.. (2020). International Olympic Committee Consensus Statement: Methods for Recording and Reporting of Epidemiological Data on Injury and Illness in Sports 2020 (Including the STROBE Extension for Sports Injury and Illness Surveillance (STROBE-SIIS)). $\mathrm{Br}$ J Sport Med, (54), 372-389. https://doi.org/10.1136/bjsports-2019-101969

Baron, D. A., Baron, S. H., Tompkins, J., \& Polat, A. (2013) Assessing and treating depression in athletes. In D. A. Baron, C. L. Reardon, \& S. H. Baron (Eds.), Clinical sports psychiatry: An international perspective (pp. 65-78). West Sussex: Wiley-Blackwell.

Beable, S., Fulcher, M., Lee, A. C., \& Hamilton, B. (2017). SHARPSports mental Health Awareness Research Project: Prevalence and risk factors of depressive symptoms and life stress in elite athletes. Journal of Science and Medicine in Sport, 20(12), 1047-1052.

Bech, P., Rasmussen, N. A., Olsen, L. R., Noerholm, V., \& Abildgaard, W. (2001). The sensitivity and specificity of the Major Depression Inventory, using the Present State Examination as the index of diagnostic validity. J Affect Disord. 66(2-3):159-164.

Bech, P., Timmerby, N., Martiny, K., Lunde, M., \& Soendergaard, S. (2015). Psychometric evaluation of the Major Depression Inventory (MDI) as depression severity scale using the LEAD (Longitudinal Expert Assessment of All
Data) as index of validity. BMC Psychiatry, 15(1). https://doi.org/10.1186/s12888-015-0529-3

Beck, A.T., Ward, C. H., Mendelson, M., Mock, J., \& Erbaugh, J. (1961) An inventory for measuring depression. Archives of General Psychiatry, 4, 561-571

Bissett, J. E., Kroshus, E., \& Hebard, S. (2020). Determining the role of sport coaches in promoting athlete mental health: a narrative review and Delphi approach. BMJ Open Sport \& Exercise Medicine, 6(1), 1-9.

Breslau, J., Gilman, S.E., Stein, B.D., Ruder, T., Gmelin, T., \& Miller, E. (2017). Sex differences in recent first-onset depression in an epidemiological sample of adoles- cents. Translational Psychiatry, 7(5), e1139-e1139. PubMed ID: 28556831 https://doi.org/10.1038/tp.2017.105

Bryman, A. (2012). Social research methods (4th ed.). Oxford: Oxford University Press.

Castaldelli-Maia, J. M., e Gallinaro, J. G. de M., Falcão, R. S., Gouttebarge, V., Hitchcock, M. E., Hainline, B., ... Stull, T. (2019). Mental health symptoms and disorders in elite athletes: a systematic review on cultural influencers and barriers to athletes seeking treatment. British Journal of Sports Medicine, 53(11), 707-721.

Cuijpers, P., Boluijt, P., \& Van Straten, A. (2008). Screening of depression in adolescents through the Internet. European Child \& Adolescent Psychiatry, 17(1), 32-38.

DeVellis, R. F. (2017). Scale development: Theory and applications (4th ed.). Los Angeles: SAGE.

Fernandes, G. S., Parekh, S. M., Moses, J., Fuller, C. W. Scammell, B., Batt, M. E., ... Doherty, M. (2019). Depressive symptoms and the general health of retired professional footballers compared with the general 
population in the UK: a case-control study. $B M J$ Open, 9(9), e030056.

Golding, L., Gillingham, R. G., \& Perera, N. K. P. (2020). The prevalence of depressive symptoms in high-performance athletes: a systematic review. The Physician and Sportsmedicine, 1-12. https://doi.org/10.1080/00913847.2020.1713708

Gorczynski, P. F., Coyle, M. \& Gibson, K. (2017). Depressive symptoms in high-performance athletes and non-athletes: a comparative meta-analysis. Br J Sports Med, 51, 13481354. https://doi.org/10.1136/bjsports-2016-096455

Gouttebarge, V., Frings-Dresen, M., \& Sluiter, J. (2015). Mental and psychosocial health among current and former professional footballers. Occupational Medicine, 65(3), 190-196. https://doi.org/http://dx.doi.org/10.1093/occmed/kqu202

Hammond, T., Gialloreto, C., Kubas, H. \& Hap Davis, H. (2013) The prevalence of failure-based depression among elite athletes. Clin J Sport Med, 23(4), 273-277. https://doi.org/10.1097/JSM.0b013e318287b870

Hankin, B. L. (2012). Future directions in vulnerability to depression among youth: Integrating risk factors and processes across multiple levels of analysis. Journal of Clinical Child and Adolescent Psychology, 41(5), 695-718. https://doi.org/10.1080/15374416.2012.711708

Henriksen, K., Schinke, R., Moesch, K., McCann, S., Parham, W. D., Larsen, C. H. \& Terry, P. (2019). Consensus statement on improving the mental health of high performance athletes. International Journal of Sport and Exercise Psychology, 1-8. https://doi.org/10.1080/1612197X.2019.1570473

Jensen, S. N., Ivarsson, A., Fallby, J., Dankers, S., \& Elbe, A.-M. (2018). Depression in Danish and Swedish elite football players and its relation to perfectionism and anxiety. Psychology of Sport \& Exercise, 36, 147-155.

Kuettel, A., Pedersen, A. K., \& Larsen, C. H. (2020). To flourish or languish, that is the question: Exploring the mental health profiles of danish elite athletes. Psychology of Sport and Exercise,

101837. https://doi.org/10.1016/j.psychsport.2020.10183 7

Kuettel, A. \& Larsen, C. H. (2019). Risk and protective factors for mental health in elite athletes: a scoping review. International Review of Sport and Exercise Psychology. https://doi.org/10.1080/1750984X.2019.1689574

Larsen, C. H., Kuettel, A., Moesch, K., Durand-Bush, N., \& Henriksen, K. (2021). Setting the scene: Mental health in elite sport. In C. H. Larsen, K. Moesch, N. Durand-Bush, \& K. Henriksen (Eds.), Mental health in elite sport: Applied perspectives from across the globe (1. ed.). Routledge. https://www.routledge.com/Mental-Health-in-Elite-SportApplied-Perspectives-from-Across-the-Globe/LarsenMoesch-Durand-Bush-Henriksen/p/book/9780367427689

Lichtenstein, M. B., Gudex, C., Andersen, K., Bojesen, A.B., \& Jørgensen, U. (2017). Do exercisers with musculoskeletal injuries report symptoms of depression and stress? Journal of Sport Rehabilitation. 5, 1-22. https://doi.org/1123/jsr.2017-0103

Lichtenstein, M. B. Melin, A., Szabo, A., Holm, L. (2021): The prevalence of exercise addiction symptoms in elite athletes. Frontiers in Sports and Active Living - Movement Science and Sport Psychology. In Press.

Lichtenstein, M. B., Nielsen, R.O., Gudex, C., \& Jørgensen, U (2018). Exercise addiction is associated with emotional distress in injured and non-injured regular exercisers. Addictive Behaviors Report, 8, 33-39.

Manea, L., Gilbody, S., \& McMillan, D. (2015). A diagnostic metaanalysis of the Patient Health Questionnaire-9 (PHQ-9) algorithm scoring method as a screen for depression. General Hospital Psychiatry, 37(1), 67-75. https://doi.org/10.1016/j.genhosppsych.2014.09.009

Niebauer, J., Boerjesson, M., Carre, F., Caselli, S., Palatini, P., Quattrini, F., ... Pelliccia, A. (2018). Recommendations for participation in competitive sports of athletes with arterial hypertension: a position statement from the sports cardiology section of the European Association of Preventive Cardiology (EAPC). European Heart Journal, 39(40), 3664-3671.
Purcell, R., Gwyther, K., \& Rice, S. M. (2019). Mental health in elite athletes: increased awareness requires an early intervention framework to respond to athlete needs. Sports Medicine-Open, 5(1), 1-8.

Radloff, L. S. (1977). The CES-D scale: A self-report depression scale for research in the general population. Applied Psychological Measurement, 1(3), 385-401.

Reardon, C. L., Hainline, B., Aron, C. M., Baron, D., Baum, A. L., Bindra, A., ... Engebretsen, L. (2019). Mental health in elite athletes: International Olympic Committee consensus statement (2019). British Journal of Sports Medicine, 53(11), 667-699. https://doi.org/doi:10.1136/bjsports2019-100715

Rice, S. M., Gwyther, K., Santesteban-Echarri, O., Baron, D., Gorczynski, P., Gouttebarge, V., ... Purcell, R. (2019). Determinants of anxiety in elite athletes: a systematic review and meta-analysis. British Journal of Sports Medicine, 53(11), 722-730.

Rice, S. M., Purcell, R., De Silva, S., Mawren, D., McGorry, P. D., \& Parker, A. G. (2016). The mental health of elite athletes: a narrative systematic review. Sports Medicine, 46(9), 1333-1353.

Salk, R. H., Hyde, J. S., \& Abramson, L. Y. (2017). Gender differences in depression in representative national samples: Meta-analyses of diagnoses and symptoms. Psychological Bulletin, 143(8), 783-822.

Schaal, K., Tafflet, M., Nassif, H., Thibault, V., Pichard, C., Alcotte, M., ... Toussaint, J.-F. (2011). Psychological balance in high level athletes: gender-based differences and sportspecific patterns. PloS One, 6(5), 1-9. https://doi.org/10.1371/journal.pone.0019007

Spitzer, R. L., Kroenke, K., Williams, J. B. W., \& Löwe, B. (2006). A brief measure for assessing generalized anxiety disorder: the GAD-7. Archives of Internal Medicine, 166(10), 1092-1097.

Sundgot-Borgen J, \& Torstveit M.K. (2004). Prevalence of eating disorders in elite athletes is higher than in the general population. Clin J Sport Med. 14(1), 25-32. https://doi.org/10.1097/00042752-200401000-00005

Szabo, A., De laVega, R., Ruiz-Barquín, R., Rivera, O. (2013). Exercise addiction in Spanish athletes: Investigation of the roles of gender, social context and level of involvement. $J$. Behav. Addict. 2(4), 249-52.

Tahtinen, R., Kristjansdottir, H., Olason, D. T., \& Morris, R. (2021). What lies beneath: exploring different depressive symptoms across selected risk factors in Icelandic team sport athletes. Journal of Clinical Sport Psychology. https://doi.org/10.1123/jcsp.2020-0040

Tahtinen, R. E., Shelley, J. \& Morris, R. (2021). Gaining Perspectives: A Scoping Review of Research Assessing Depressive Symptoms in Athletes. Psychology of Sport and Exercise, 101905. https://doi.org/10.1016/j.psychsport.2021.101905

Weekendavisen (2020). Accessed 6/10/2021 from https://www.weekendavisen.dk/2020-21/ideer/sundtlegeme-sund-sjael 